\title{
El Desarrollo Profesional Docente: diario pedagógico y práctica
}

\section{Teaching Professional Development: pedagogical and practical journal}

https://doi.org/10.26852/2357593X.193

\author{
Armando Zambrano Leal ${ }^{2}$
}

\section{Resumen}

En las últimas tres décadas el ethos de la institución universitaria cambió radicalmente. Después de las reformas de Bolonia y de Paris, la universidad entró en una nueva lógica, nuevas demandas, nuevas realidades cuyo objetivo fundamental son las condiciones de formación del estudiantado, sus aprendizajes y la evaluación. El docente, en los nuevos escenarios de la universidad, toma un papel relevante para los procesos de calidad. De sus niveles de formación, capacidades de investigación, extensión y gestión del conocimiento, depende, en gran parte, la calidad de la institución. Todos los insumos de calidad, finalmente, se dirigen a su profesionalidad. Las buenas prácticas didácticas determinan excelentes resultados en los aprendizajes del estudiantado y aquí surge un nuevo desafío institucional: observar las prácticas para mejorar las disposiciones del profesorado. Este desafío está estrechamente asociado con el Desarrollo Profesional Docente. Pues bien, este artículo de reflexión se centra en el concepto de DPD, las prácticas y el dispositivo de formación conocido como "diario pedagógico”.

Palabras Clave: [Plan de carrera], [Profesión docente], [Estilo de enseñanza], [Prácticas didácticas], [Innovación pedagógica].

\section{Abstract}

In the last three decades the ethos of the university institution changed radically. After the Bologna and Paris reforms, the university entered a new logic, new demands, new realities whose main objective is the conditions of student training, their learning and evaluation. The teacher, in the new settings of the university, takes a relevant role for quality processes. The quality of the institution depends to a large extent on its levels of training, research capacities, extension and knowledge management. All the quality inputs, finally, are directed to your professionalism. Good didactic practices determine excellent results in student learning and here a new institutional challenge arises: observing practices to improve the dispositions of teachers. This challenge is closely associated with Teacher Professional Development. Well, this article for reflection focuses on the concept of PD, the practices and the training device known as the "pedagogical diary".

Keywords: [Career plan], [Teaching profession], [Teaching style], [Didactic practices], [Pedagogical innovation].

\footnotetext{
1 El presente artículo de reflexión toma como base la propuesta de intervención en Desarrollo Profesional Docente en la Fundación Universitaria Sanitas. El texto ha sido ampliado para efectos de la publicación en esta Revista.

2 Licenciado en Ciencias de la Educación, Universite Lumiere Lyon, Francia. Mg. Ciencias de la educación, Universite Lumiere, Francia. Doctor Sciences de l'éducation, Université Paris, Francia. Profesor Universitario.

Citar como: Zambrano,L,(2019). El Desarrollo Profesional Docente: Diario Pedagógico y Práctica. Revista de desarrollo profesoral, Volumen No. 7 , pag. 92-100. https://doi.org/10.26852/2357593X.193
} 


\section{El Desarrollo profesional docente}

\section{Génesis y variantes}

La historia del Desarrollo Profesional Docente (DPD) tiene sus inicios a mediados de 1980 con el nacimiento, en Estados Unidos, del movimiento denominado career ladder el cual puede traducirse como plan de carrera. Este movimiento es producto de la reforma adoptada, en 1984, por el Estado de Tennesse y dirigido a mejorar la profesión docente. Tres años después, 31 Estados habían adoptado el término Plan de Carrera cuyos objetivos consistían en mejorar los salarios sobre la base del rendimiento y la promoción producto de una evaluación, salarios diferenciados y desarrollo profesional docente (Barnabé, 1993). Los planes de carrera definieron una jerarquización de las actividades del docente y una diferenciación de competencias lo que permitía la promoción y movilidad al interior de la profesión.

El DPD parte de dos grandes premisas: ¿¿De qué manera pueden ser mejorados los procesos de enseñanza y aprendizaje? ¿Cómo tantos profesores pueden trabajar en dicha mejora? (Ruè, 2015: 221). Estas dos cuestiones están en la base de las mejoras de la enseñanza, tanto en la educación escolar como en la superior y es el camino considerado como el más adecuado para potenciar la enseñanza y los aprendizajes (Vaillant y Cardozo, 2016). El DPD indiscutiblemente está siuado en el registro de los aprendizajes de los estudiantes. Este recibe diversos nombres: aprendizaje por problemas, formación continua, formación profesional situada (Duque, Celis, Diaz, y Gómez, 2014). Así mismo, existen diversos dispositivos de DPD clasificados en institucionales y colectivos-personales. Los primeros son agenciados por las instituciones (escolares o universitarias) y los segundos, por comunidades de aprendizaje en sus diversas manifestaciones : grupos de estudio, grupos de investigación-acción, grupos de desarrollo pedagógico (Dionni, Lemyre. Savoie-Zajc, 2010).

El DPD integra dos elementos claves: el desarrollo como evolución y continuidad (Vaillant, 2016) y el profesional que implica la identidad de la función. El desarrollo supone, también, cambio en las prácticas.
Pero ¿qué si significa cambiar en una práctica?. Lo que sostienen (Deaudelin, Brodeur, \& Bru, 2005) es precisamente que los dispositivos desplegados a través de la investigación sobre DPD se dirigen a intervenir en los cambios tanto de las representaciones como en las acciones que emprenden los docentes en su ejercicio profesional. Estas representaciones son individuales y colectivas y se movilizan a través de la acción institucional pues ella recoge y organiza las disposiciones sociales y estatales sobre la calidad educativa.

En general, el DPD se define como el aprendizaje realizado por el docente, a la vez como un proceso y producto. Esto implica que dicho desarrollo sería la base para dos grandes corrientes: la desarrollista y la profesionalizante. En el primer caso, el desarrollo se estima como crecimiento de los actores e implica modificaciones en sus acciones, sentimiento a lo largo del tiempo. Esta perspectiva se apoya en los mecanismos piagetianos de modificación y acomodación. Desde la perspectiva de la profesión, se insiste en el perfeccionamiento a través de la formación continua, los procesos de aprendizaje a lo largo de la vida, las diversas formas de aprendizaje, investigación continua y reflexión sobre la práctica, tranformación de las competencias y componentes identitarios y toda experiencia de aprendizaje natural las que generalmente se impulsan a través de las instituciones y que corresponden con el cambio de linealidad en el empleo por el de fluctuación de las experiencias. (Uwamariya \& Mukamurera, 2005), (Brodeur, Monique. Daudelin, Colette \& Bru, Marc, 2005).

En este orden, el DPD es un proceso por medio del cual el profesor y sus colegas revisan y renuevan conjuntamente, como agentes de cambio, adquieren y desarrollan sus conocimientos, las habilidades y saberes para un buen ejercicio profesional (Day, 1999). También significa una diversidad de aprendizajes en la cual los profesores se comprometen, plantean soluciones y conciben nuevas prácticas de su actividad. En el fondo, el DPD hace de la práctica su terreno de acción lo que impone conceptualizarla más allá del simple hacer. 


\section{Desarrollo profesional docente y práctica}

En el campo de la formación docente, desde la década de 1970, tomó mucha fuerza el "análisis de necesidades", técnica proveniente de la formación de adultos y aplicada, en múltiples investigaciones, a la formación docente (Lang, 1987). Luego, emergerá el concepto de la práctica, como análisis de prácticas, en la cual se trataba de comprender la acción y los saberes fundamentales del docente. Mientras el análisis de necesidades imponía la idea de "faltante", "carencia", "demanda", el de práctica situaba la reflexión, los dispositivos, los saberes en la acción y la experiencia docente como fuente de identidad. En esta década se dejó de lado el "análisis de necesidades" pues no se trataba de suplir la carencia en la profesión sino de investigar el hacer en la profesión, el cómo se hacía. La práctica aquí quedó reducida a la acción y dio origen al concepto de "práctica pedagógica". La práctica también se empobreció pues muchos la utilizaron como el conjunto de actividades que los profesores realizan en la enseñanza y era contraria a teoría. Observar las prácticas se volvió un asunto clave para el mejoramiento docente. Suponía esta perspectiva que el docente era un intelectual de la clase y que la manera como lo hacía marcaba el resultado final. En últimas, observando las prácticas se deducían los resultados del sistema pues el profesor era responsable de la buena enseñanza y de los logros escolares; interviniendo en ellas, todo mejoraría.

Este concepto abrió el espectro de su conceptualización y no sólo por la acción directa sino también por la historia de la institución, los sujetos, los saberes. Por ejemplo, a través del estudio de las prácticas pedagógicas en una época se deducía el ideal de educación de dicha época. También las prácticas han servido para comprender los ideales filosóficos de la educación e incluso sociológicos y económicos. Esta orientación parte del presupuesto de que la práctica remite a la acción humana -educativa y se opone a la teoría, un conjunto de percepciones, de pensamiento, de acción y de evaluación que nos guía (Noël, 1997). La práctica está nutrida por los cambios en las representaciones y la construcción de modelos persona- les para actuar en el aula, promover los aprendizajes, formarse así mismo. (Baillauquès, 1988). Es en este orden que ellas dieron lugar al análisis de prácticas por medio del cual se buscaron conocer los modelos, las formas, las estrategias, las relaciones entre sujetos, la innovación, las relaciones con el conocimiento de los docentes (Altet, 2000).

Pues bien, la práctica tiene tres grandes registros. Las disposiciones, el modo y el estilo (Zambrano, 2007). Las primeras tienen que ver con el deseo de hacer, realizar la actividad, tener placer y sentir gusto. Es decir, cuando alguien se dispone plenamente realiza la actividad de forma creativa y encuentra en tal hacer un sentido para su vida. Al contrario, cuando no existe disposición, la actividad ni produce placer ni deseo y menos gusto. En la acción de un proceso repetitivo, generalmente en los obreros y operarios, las acciones terminan siendo aburridas $y$, muchas veces o con frecuencia, la persona siente muy poco placer y gusto por la actividad; la resignación es su sello. En el caso contrario, por ejemplo, el del artista o el del artesano, placer, deseo y gusto afloran como disposición pues creando el sujeto enriquece su vida, su espíritu. El artista genial expresa la emoción en su obra y ella contiene todo el placer, el gusto, lo creativo. En la fina artesanía se observa el placer, el gusto, la creatividad del artesano. Ahora bien, el modo de llevar a cabo una actividad engrandece el espíritu de quien la realiza.

El modo de organizar la clase, los materiales, los procedimientos, las situaciones de aprendizaje denotan madurez, desarrollo, plenitud. El modo es producto de la experiencia y es un saber que emerge gracias a la disposición. Finalmente, el estilo es el diferenciador de la actividad y es la finitud de la acción creativa; se alcanza por la disposición y el modo. El estilo marca la diferencia entre el experto y el novato, entre la pasión y la obligación, entre la creatividad y la repetición. El estilo de enseñanza es la finura de la acción y muestra el pleno desarrollo de la formación, la altura alcanzada, la plenitud. El estilo en el artista como en el artesano es el signo 
diferenciador de su creatividad. El estilo es el conjunto de gestos que dan cuenta de la perfectibilidad alcanzada. En el caso del profesorado, el estilo muestra su identidad, el desarrollo tanto de sus gestos como de sus pensamientos y a través de ellos se observa el modo cómo realiza la actividad y, finalmente sus disposiciones. Estos tres elementos son la base fundamental de la práctica y, por esto mismo, ella no es un simple hacer.

El DPD apunta a mejorar las competencias de los profesores noveles a través de diversas estrategias, por ejemplo el de la tutoría de pares (Moussay, Méard \& Étienne, 2011), la observaciónde clases, las comunidades de aprendizaje, etc; entonces, es muy importate registrar la disposición, el modo y estilo como el profesorado asume su actividad y esto porque los aprendizajes de los profesores se sitúan en dos registros: el desarrollo de las competencias (saberactuar) en situaciones complejas que exigen dos condiciones: saberes procedimentales (teóricos y procedimentales) y la facultad para mobilizarlos, resolver problemas y tomar decisiones (Perrenoud. , 2000). La autoregulación de los aprendizajes aparece como el mecanismo de los cambios en la educación. En las instituciones se acrecienta el interés de hacer aprender a los estdiantes. Ello implica, tal como lo hemos visto, mejorar la práctica lo que impone el autoraprendizaje, trabajo autónomo, aprendizaje autónomo, etc. La autoregulación del aprendizaje es objeto de investigación sobre los formadores de docentes (Tillema, 2005) y esto es un asunto de práctica.

\section{¿Por qué observar la práctica?}

La observación ha tenido tres grandes paradigmas. El prescriptivismo y su modelo de enseñanza eficaz; el modelo cognitivista sobre el control de la práctica de enseñanza en cuanto el profesor es visto como alguien que decide, a partir de sus pensamientos, teorías y opiniones personales, la forma como planifica sus acciones y las pone en práctica en el aula y, finalmente, el paradigma "ecologista" en el cual se privilegia la "situación" como variable clave de la práctica de enseñanza. Más reciente, el modelo interaccionista donde se integran variables personales, procedimentales y contextuales y no se centra exclusivamente en el profesor sino también en el alumno en cuanto su mirada y puntos de vista ayudan a comprender mejor la práctica del docente y mejorar, en estado de acción crítica, el desarrollo profesional docente.

Desde inicios de la década de los años 80 del Siglo anterior, las investigaciones muestran una gran preocupación por observar las clases más como un medio para comprender los modos como un profesor enseña, se forma, aprende de las situaciones vividas en clase y generar conocimiento de su práctica y menos como un dispositivo de corrección. (Altet, 2017) señala tres grandes tipos de observación: declaradas, constatadas y esperadas. Las primeras se refieren a lo que dice hacer el sujeto profesor, las segundas las que espera el profesor que le brinde la institución y las terceras las que observa el investigador en la actividad que despliega el profesor en situación de clase. Los instrumentos de observación son variados. Para la observación, por ejemplo, se privilegian las encuestas, cuestionarios o entrevistas.

La observación va más allá de la simple mirada escrutadora, es una instancia de reflexión en común. Se trata de un trabajo entre pares quienes, en comunidad, se nutren de los relatos individuales. Las voces individuales informan de la experiencia y con ella, de los obstáculos, las contingencias, las deficiencias o los avances. Toda situación vivida en clase deviene un importante laboratorio de la experiencia y una fuente suprema de textualidad. Los momentos de la clase son conjuntos de instantes por los que transita el profesorado, ellos están cargados de símbolos a los cuales hay que encontrarles un sentido. Se observa la situación y esto implica partir del relato de lo cotidiano, de vivido y de lo concebido. Este relato es portador de valor y contribuye en la identidad del profesorado. Así mismo, esta textualidad da cuenta de la relación con el saber del profesorado. La relación con el saber aquello que la práctica une con lo pedagógico y que permite explicar las relaciones con la disciplina, el mundo, los otros, consigo mismo. 


\section{¿Qué se observa?}

Observar es encontrar el sentido a lo concebido, vivido, dado en la situación y, en este caso, a la situación de clase. ¿Qué se observa? Todo el itinerario que va desde la relación con el saber del profesor (su formación en la disciplina), la forma de planificar u organizar el contenido de su saber (enseñado), el modo como lo pone en escena, en y fuera del aula (saber de acción), la manera como acompaña el aprendizaje (saber práctico), el momento como evalúa y hace uso de ella para el aprendizaje exitoso (saber de distancia), la producción del saber dado en forma de saber exteriorizado (investigación sobre su práctica).

El itinerario de la observación está estrechamente unido a la situación de clase. Aquí es muy importante la formación en didáctica de su disciplina o didáctica específica. La historia y epistemología de la didáctica nos muestra, en la perspectiva francófona, que ella es una disciplina cuyo objeto de estudio es la génesis, circulación y apropiación del saber (enseñado) y sus condiciones de enseñanza y aprendizaje (Zambrano; 2005). Formar a los profesores en didáctica es más que un recurso meramente técnico. Se trata de acercarlos y ayudarles a formarse en esta disciplina pues ella es la que mejor ha operado el sistema del aprendizaje. La didáctica tiene al aprendizaje por objeto y se interesa en las condiciones para que tenga lugar en los estudiantes. Ahora bien, la didáctica se específica en orden al objeto de la disciplina que estudia, organiza, regula en términos de aprendizaje. Por esto, es necesario aprender los fundamentos de la didáctica (¿general?) como teoría y las didácticas específicas o disciplinares. Así, entonces, el profesor debe dominar la didáctica de la medicina, la enfermería, la psicología. En esto hay grandes avances pues hace ya cerca de una década se prioriza, en el campo universitario, la didáctica para las disciplinas como espacio de desarrollo profesional docente (DPD). Ahora bien, es necesario afirmar que el cambio en las prácticas docentes está fuertemente relacionado con la innovación pedagógica. ¿A que llamamos Innovación pedagógica?

\section{Innovación pedagógica}

El tema de la innovación pedagógica en la universidad es muy reciente y está fuertemente arraigado a los cambios de la Universidad y sus prácticas de formación. Este concepto viene de la empresa, se consolida en la tecnología y se desplaza a la educación en la primera década de presente siglo. Una investigación sobre el tema realizada, entre otros por (Béchard, 2001) muestra la usencia de trabajo en esta línea antes del año 2000. (Fichez, 2008) mostraba la evolución del tema en los aspectos curriculares y en la pedagogía universitaria. La profesionalización de la enseñanza universitaria ha tomado un lugar importante en la pedagogía universitaria. Esto significa que los profesores no sólo deben comprometerse con la investigación, sino que también deben desarrollar habilidades pedagógicas con el fin de promover prácticas eficaces y cambios importantes en la enseñanza (Lison \& Jutras, 2014). En el marco de la profesionalización del docente universitario se encuentra la innovación. Esta es, desde cualquier punto de vista, una "transgresión" de las reglas establecidas, de las formas de hacer y representa una forma de irrupción de lo establecido y siempre por mejorar (Coulibay \&Hermann, 2015). La innovación es un proceso contrario a novación, cambio, renovación o reforma.

No todo cambio es innovador, pero si toda innovación produce cambios. La innovación designa un proceso y sus resultados. En su etimología, innovar es "introducir algo nuevo en" y esto representa introducir algo nuevo en un contexto. Lo que es nuevo en la innovación es la cosa que cambia en el contexto, mejorar lo que existe, lo que se percibe. En pedagogía universitaria, se considera innovación aquello que sobrepasa la cátedra magistral. Por ejemplo, la adopción del aprendizaje por problemas en la facultad de medicina de Sherbrooke en 1987 rompió con la cátedra tradicional e implicó un cambio en la organización de los aprendizajes tal como lo plantearon los creadores de este enfoque en la Universidad de Mcmaster y así se fue extendiendo a otras universidades como Limburg en Maastrich (Holanda), New Castle (Australia), en la Universidad de Bourgogne (Francia) o en Louvaine (Bélgica). Este nuevo enfoque rompe con la tradición de la enseñanza de la 
medicina, ingresa como algo nuevo en un contexto de tradición y, es considerada, entonces, como innovación de aprendizaje. (Lison, Bédard, Beaucher \& Trudelle, 2014).

La innovación va dirigida al programa, los enfoques pedagógicos, los procesos de transmisión de aprendizaje, los sujetos profesores y estudiantes, los mecanismos de evaluación. (Kozanitis, 2010). Hay factores que favorecen o inhiben la innovación pedagógica: a). las personas que se comprometen con la innovación pedagógica deben ser conscientes del impacto que tendrá sobre ellas y sobre los otros. Para lograrlo, deben establecer los valores; b) la persona que innova es vista como creativa, capaz de iniciativa y de riesgos y c) tomar el tiempo de la innovación con paciencia pues ella exige ir con calma ya que lo que se innova son las prácticas (Bédard, \& Raucent, 2015).

\section{El diario pedagógico: práctica e innovación}

Como lo hemos visto, la práctica no es un simple hacer. Ella implica tres conceptos fundamentales: disposición, modo y estilo; estos tres elementos la sacan del reduccionismo tecnicista y la ponen en un horizonte de creatividad. Así, en el DPD, la práctica es una instancia de reflexión pedagógica y didáctica fuertemente ligada a la innovación. Uno de los medios eficaces para reflexionar la práctica es el diario pedagógico. Este se entiende como un dispositivo proveniente de la etnosociología y de las corrientes autobiográficas que nacen de las historias de vida. La palabra diario, en francés, está ligada a la práctica de escritura y a lo cotidiano donde se germinan las diversas acciones de lo humano. (Hess, 1998). También se remonta a la historia del diario familiar y cuyos vestigios muestra muy bien Frédéric Le Play a través de, por ejemplo, obras como: La vie domestique, ses modèles et ses règles, d'après des documents originaux (La vida cotidiana, sus modelos y sus reglas a partir de documentos originales). Una famille au XVle siècle (Una familia en el siglo XVI), ilustra la práctica del diario de las personas en el siglo XVI. Esta práctica se conoció como diarios de razón, donde las familias apuntaban con pulcritud lo cotidiano de sus vidas.
Desde allí nace el movimiento conocido como diarismo el cual es un método de gestión del tiempo y de organización de la vida. Esta práctica fue reconocida como ensayo, entró plenamente en 1808 como instrumento educativo cuya finalidad consistía en un medio organizador de la vida de los jóvenes en el plano moral, intelectual y físico. Dicha práctica la encontraremos, igualmente, en Marc-Antoine Jullien y sus Ensayo sobre el empleo del tiempo. (1808-1810). Fue Jullien quien avanzó en un método del diario con las siguientes fases: i). primero, aprender a preguntarse antes de hablar o actuar; ii) al finalizar cada jornada (mañana o noche) hay que evaluar cómo la jornada fue empleada tanto a nivel del corazón, el espíritu o el cuerpo; iii) no hay que limitarse a un acto puramente de meditación y de reflexión sino detenerse en los logros de la jornada. Este método producirá, según JuIlien, doce grandes hábitos: 1). No hacer algo que entristezca nuestro temperamento y sujetarse a un régimen los más adecuado posible para la salud. 2). Vigilarse a sí mismo. 3). Superar los propios defectos. 4). Estudiar y conocer a los hombres. 5). Escoger bien a sus amigos y de preferencia a quienes más lo instruyen a uno. 6). Aprender de todas las personas en función de nuestro propio perfeccionamiento. 7). Hablar poco, saber callar y guardar un secreto. 8). Observar y reflexionar, madurar la razón y aprender de las experiencias y conocimientos de otros. 9). Ejercitar la memoria. 10). Analizar con precisión. 11). escribir con facilidad y formar, a la vez, su propio juicio y estilo. 12). Apreciar el empleo del tiempo, vivir mucho más que el resto de aquellas personas, quienes pierden con frecuencia una gran cantidad de horas y de años de su vida en cosas innecesarias. (Illiade, 2006::44).

Pues bien, como lo muestran los doce hábitos de Jullien, se convertirá en un método de reflexión de lo cotidiano en todos los aspectos de la vida. Es en esta perspectiva que el diario pedagógico, como técnica de saber y de análisis de la vida cotidiana, será recogido por los pedagogos institucionalistas a partir de 1960. Ellos lo introducirán para reflexionar la gestión en la clase y las prácticas de los profesores. Con este dispositivo, los profesores pueden reflexionar sus prácticas en términos de disposiciones, modos y estilos de transmisión del saber. 
En las disposiciones se trata de apuntar las motivaciones, frustraciones, deseos, placer, gustos, etc., frente a una temática que ha de enseñarse. El modo, es decir, la manera como el sujeto profesor transmite el saber y que encuentra un fuerte apoyo en la disposición será clave para la mejora en la forma de organizar la clase, los momentos que allí se viven, los silencios como las reacciones de los estudiantes y del mismo profesor. Finalmente, el estilo es algo que se aprende con los años pero que exige ser pulido, labrado, trabajado, experimentado, reflexionado. El diario pedagógico le permitirá al profesorado reflexionar, con los años, el estilo de su enseñanza, el modo como lo hace y sus disposiciones también para aprender. Estos tres elementos de la práctica son claves en el Desarrollo Profesional Docente.
Digamos que el diario pedagógico, utilizado en amplios sectores del profesorado universitario y escolar, es una técnica de reflexión de la práctica y un medio de innovación pedagógica. Cuando el profesorado escribe la cotidianidad de sus clases encuentra allí insumos de reflexión que lo formarán como profesor, le ayudarán a crecer y a comprender sus propias limitaciones. También, el diario, es un valioso insumo de escritura para todo aquel que quiera investigar sus propias prácticas, la vida en la institución, la cotidianidad de los otros colegas en la institución, los ambientes y haciéndolo, logrará narrar sus propias creencias, saberes y disposiciones. El diario es un elemento clave de la innovación pedagógica porque en el se incuban las ideas provenientes de la práctica. En esto, el diario pedagógico es un valioso insumo de formación profesional docente y un importante mecanismo de escritura.

\section{Conclusiones}

El EPD, como lo hemos visto, inicia como un plan de carrera y luego se transforma en un dispositivo eficaz de formación y autoformación. También permite la gestación, desarrollo y consolidación de comunidades de aprendizaje. La identidad del profesorado no depende exclusivamente de factores exógenos como podría pensarse en el plan de carrera (mejores salarios, por ejemplo), sino de una práctica reflexiva individual y colectiva Por ello, el concepto de práctica es tomado desde la perspectiva crítica más que determinista, pues en ella el profesorado encuentra los insumos de su identidad docente gracias a la reflexión sobre sus disposiciones, modos y estilos de enseñanza. Otro elemento clave es el de innovación, el cual, como lo hemos visto, está fuertemente ligado al ejercicio de creatividad pedagógica para hacer que los estudiantes aprendan mejor. Pero, para que esto suceda, el profesorado debe contar con insumos de reflexión sobre su cotidianidad profesional y uno de estos insumos es el diario pedagógico.
El diario es una práctica de sí, un modo de relatar en silencio y para sí lo vivido en lo cotidiano. Este dispositivo es muy útil a la hora de mejorar las prácticas pedagógicas y didácticas en clase. Sus efectos son numerosos y muy positivos: genera una práctica de escritura de la realidad institucional, le ayuda al profesorado a detenerse en los momentos positivos y negativos de la enseñanza, contribuye como distanciador en su reflexión pedagógica, didáctica y formativa, es generador de identidad y, lo más importante, propicia en el profesorado una práctica de escritura tan necesaria en tiempos de competitividad universitaria. En cuanto a la identidad, asunto clave en el DPD, el diario pedagógico forja en el sujeto profesor elementos de consciencia sobre su práctica y nutre el diálogo con los colegas. 


\section{Referencias}

Altet, M. (2000). L’analyse des pratiques: une démarche de formation. Recherche \& Formation (35), 25-41.

Altet, M. (2017). L'observation des pratiques enseignantes effectives en classe: Recherche et Formation. Revue Cardernos de Pesquisa, 47 (16), $1196-$ 1223.

Baillauquès, S. (1988). La question du modèle dans le discours des instituteurs. Recherche \& Formation(4), 23-36.

Barnabé, C. (1993). Un plan de carrière pour les enseignants: léxpérience américaine. Revue de Sciences de l'éducation, 18(1), 17-28.

Béchard, J.-P. (2001). L'enseigneent supérieur et les innovations pédagogiques: une recension des écrits. Sciences de l'éducation, 27(2), 257-281.

Bédard, Denis \& Raucent, Benoît. (2015). Les innovations pédagogiques en enseignement supérieur: pédagogies actives en présentiel et à distance. Revue International de Pédagogie de l’Enseignement Supérieur, 31(1), En línea.

Brodeur, Monique. Daudelin, Colette \& Bru, Marc. (2005). Le développement professionel des enseignants: apprendre à enseigner pour soutenir l'apprentissage des élèves. Revue des Sciencies de l'Éducation, 31(1), 5-14.

Coulibay, Bernard \&Hermann, Hélène. (2015). L’appropiation d'une innovation par ses usagers: autoru du futur learning centre de l¿université de Haaute alsace. (e. Ligne, Ed.) Revue International de Pédagogíe de l'enseignement supérieur, 3(12).

Day, C. (1999). Developing teachers. The challenges of lifelong learning. Londres: Falmer Press.

Deaudelin, Colette. Brodeur, Monique \& Bru, Marc. (2005). Un portrait caracteristique de la recherche sur le développement professionnel des enseignants et sur la formation à l'enseignement. Revue des Sciences de l'Éducation, 31(1), 177-185.

Dionni, Liliane. Francois, Lemyre. Savoie-Zajc, Lorraine. (2010). Vers une définition englobante de la communauté d'apprentissage (CA) comme dispositif de développement professionel. Revue des Sciences de l'Education, 36(1), 25-43.

Duque, Mauricio. Celis, Jorge. Diaz, Bibiam. y Gómez, Margarita. (2014). Diez pilares para un programa de desarrollo docente centrado en el aprendizaje de los estudiantes. Revista Colombiana de Educación(67), 107-124.

Fichez, E. (2008). L'enseignement supérieur est-il contraint d'innover? Eléments d'analyse. En G. J.-D. Ficher(dir), L'université et les Tic. Chroniue d'une innovation annoncée (págs. 51-81). Bruexelles: De Boeck.

Hess, Remi. (2010). La pratique du Journal. L'enquête du quotidien. Paris, Téraèdre

Illiade, Karen. (2006) Essai sur l'emploi du temps chez M-A-J. Paris Anthropos.

Jullien, Marc-Antoine. (2006) Essai sur l'emploi du temps (1808 à 1810), Paris: Anthropos

Kozanitis, A. (2010). L'influence d'innovatios pédagogiques sur le profil motivationnel et le choix de stratégies d'apprentissage d'étudiantes et d'étudiants d'ne faculté d'ingénierie. Evue Internationelle d Pédagogie de l'énseigement Supérieur, 26(1), En ligne.

Lang, V. (1987). De l'expression des besoins à l'analyse des pratiques dans la formation des enseignants. Recherche \& Formation(2), 37-49.

Lison, Christelle \& Jutras, France. (Mai de 2014). Innover à l'Université. Penser les situations d'enseignement pour soutenir l'apprentissage. Revue Interntionale de pédagogie de l'enseignement supérieur [en línea], 30(1).

Lison, Christelle., Bédard, Denis., beaucher, Chantal \& Trudelle, Denis. (2014). De l'innovation à unmodèle dynamique innovationnnelle en enseignement supérieur. Revue Iternationale de Pédagogie de l'enseignement Supérieur, 30(1), En ligne.

Noël, J. (1997). l'analyse des pratiues éducatives. Un cadre éthique et symbolique pour éduquer le régard de l'eneignant. Recherche \& Formation(24), 49-67.

Perrenoud, P. (2001). Développer la pratiue réflexive dans le métier d'enseignant: proféssionalisation et raison pédagogique. Paris: ESF.

Le Play, Frédéric. (1976) La vie domestique, ses modèles et ses règles, d’après des documents originaux, 2 Vol, Paris Baltenweck.

Le Play, Frédéric. (1978). Une famille au XVle siècle. 1 Vol. Éditions Tours, Mame.

Ruè Domingo, J. (2015). El desarrollo profesional docente en educación superior. Agenda, referentes y propuestas para su adopción. Revista de Docencia Universitaria, 13(Número extraordinario), 2107-236. 
Tillema, H. H. (2005). Miroirs de l'autorégulation de l'apprentissage: Les dilemes des formateurs d'enseignants. Revue des Sciences de l'Éducation, 31(1), 111-131.

Vaillant, D. (2016). El fortalecimiento del desarrollo profesional docente: una mirada desde Latinoamérica. Journal of Supranational Policiesof Education(5), 5-21.

Vaillant, Denise. Cardozo Gaibisso, Lourdes. (2016). Desarrollo profesional docente: entre la proliferación conceptual y la escasa incidencia en la práctica en el aula. Cuadernos de pedagogía Universitaria, 13(26), 5-14.

Uwamariya, Angélique \& Mukamurera, Joséphine. (2005). Le développement professionnel des eneseignants: Approches théoriques. Revue des Sciencies de l'Education, 31(1), 133-155.

Zambrano Leal, A. (2005). Didáctica, pedagogía y saber. Bogotá: Magisterio.

Zambrano Leal, A. (2007). Formación, experiencia y saber. Bogotá: Editorial Magisterio 